\title{
Soft Micro- and Nanorobotics
}

\section{Review Article}

\section{Author(s):}

Hu, Chengzhi; Pané, Salvador; Nelson, Bradley

Publication date:

2018-05

\section{Permanent link:}

https://doi.org/10.3929/ethz-b-000316345

Rights / license:

In Copyright - Non-Commercial Use Permitted

\section{Originally published in:}

Annual Review of Control, Robotics, and Autonomous Systems 1, https://doi.org/10.1146/annurev-control-060117-104947

\section{Funding acknowledgement:}

743217 - Soft Micro Robotics (EC)

165564 - Soft Magnetic Robots: Modeling, Design and Control of Magnetically Guided Continuum Manipulators (SNF) 
Annu. Rev. Control Robot. Auton. Syst. 2018. 1:X-X

https://doi.org/10.1146/annurev-control-060117-104947

Copyright $@ 2018$ by Annual Reviews.

All rights reserved

Hu •Pané • Nelson

www.annualreviews.org • Soft Micro- and Nanorobotics

\title{
Soft Micro- and Nanorobotics
}

\section{Chengzhi Hu, Salvador Pané, and Bradley J. Nelson}

Institute of Robotics and Intelligent Systems, ETH Zurich, CH-8092 Zurich, Switzerland; email:

huc@ethz.ch, vidalp@ethz.ch, bnelson@ethz.ch

\section{Keywords}

soft microrobots, nanorobots, smart materials, biomimetics

\begin{abstract}
Micro- and nanorobots can perform a number of tasks at small scales, such as minimally invasive diagnostics, targeted drug delivery, and localized surgery. During the past decade, the field has been transformed in many ways, one of the most significant being a transition from hard and rigid micro- and nanostructures to soft and flexible architectures. Inspired by the dynamics of flexible microorganisms, researchers have focused on developing miniaturized soft components such as actuators, sensors, hinges, joints, and reservoirs to create soft micro- and nanoswimmers. The use of organic structures such as polymers and supramolecular ensembles as functional components has brought more complex features to these devices, such as advanced locomotion strategies and stimulus-triggered shape transformations, as well as other capabilities. A variety of microorganisms and contractile mammalian cells have also been utilized as microengines and integrated with functional synthetic materials, producing bending or deformation of the functional materials to initiate motion. In this review, we consider several types of soft microand nanorobots in terms of their architecture and design, and we describe their locomotion mechanisms and applications.
\end{abstract}

\section{INTRODUCTION}


Micro- and nanorobots is an interdisciplinary field that combines aspects of robotics, micro and nanotechnology, biomedical engineering, and materials science. (1). These devices are capable of swimming in liquid environments by harvesting fuel from their surrounding media or by harnessing power from external energy sources, such as light, ultrasound, electrical or magnetic fields, or combinations of these (2-6). Micro- and nanorobots hold promise in several applications, ranging from manufacturing, material removal, information security, and microassembly to localized diagnosis, targeted drug delivery, and implantation of active structures.

Advances in materials science and micro and nanofabrication have significantly impacted the development of micro- and nanoswimmers over the past decade. A recent example of this is the number of publications reporting on microswimmers fabricated by means of two-photon polymerization, also known as 3-D photolithography. An advantage of this approach is that it allows for the production of soft complex microarchitectures made of different photocurable polymers and polymer matrix nanocomposites. For example, Nelson and coworkers (7-9) produced several helical microswimmers and other complex designs using this manufacturing approach. In most cases, the microstructures were built by using photoresists and subsequently coated with magnetic materials for their propulsion (7-9). In other cases, a soft template can be produced using 3D photlithography and filled with other soft materials such as conductive polymers. For example, Zeeshan et al. produced microrobots consisting of a hard electroplated cobalt-nickel head with an attached electropolymerized soft polyprrole tail (10). Swimmers produced using 3-D photolithography have been used for several applications such as cargo and release of small-scale objects, therapeutic payloads and cells (11) (12) (16). In vivo tracking and magnetic steering of a swarm of helical microswimmers in a mouse body has also been recently demonstrated (15). These efforts illustrate that 3-D photolithography is a powerful tool for the fabrication of microscale swimmers with improved robotic motility for use in future biomedical robotic applications.

While there are many examples of micro- and nanoswimmers made of rigid and hard components, the use of soft materials in micro- and nanorobots is just beginning. This new area of soft micro- and nanorobotics focuses on creating swimmers from miniaturized soft materials, which include but are not limited to polymers, membranes, colloids, gels, foams, proteins, and 
microemulsions (17-21). As compared with their hard counterparts, soft robotic structures have distinct advantages: They can be manufactured using simple designs, are less dense and less costly, can be processed at lower temperatures and in aqueous environments, and in many cases exhibit a higher level of biodegradability and resorbability. Additionally, many soft materials can be highly deformed, a feature that can be exploited to exhibit new locomotion mechanisms.

The physicochemical properties of soft materials often resemble those of biological matter. Hence, the use of soft matter in small-scale robotic platforms enables the fabrication of devices that more closely mimic biological structures, including microorganisms. A distinguishing quality of many soft materials is their stimulus-responsive characteristics, which make them attractive for biomedical applications. Several polymers and supramolecular architectures respond to external triggers by changing their macroscopic shape. These materials, capable of sensing and actuation, have the potential to exhibit behavioral responses. This feature is essential from the point of view not only of functionality but also of adaptability characteristics. For instance, a small-scale device that can change its shape and adopt a different locomotion mechanism as a result of its morphological transformation can be useful in biomedical applications, where the device will face changes in the fluidic environment (i.e., the human vasculature).

Nature has designed microorganisms that can adapt their shape to accomplish their functions. For example, nematodes, which are found almost everywhere on Earth, swim in water or crawl in soil by bending their bodies as they move (22). To navigate complex fluids or fibrillar matrices, unicellular organisms either exhibit changing body forms actuated by dynamically regulated internal protein networks or use compliant appendages, such as flagella and cilia. When these organisms encounter a change in physical surrounding, the reconfigurable nature of their mechanical structure allows them to adapt their locomotive gaits. Microorganisms, including many eukaryotic and prokaryotic cells, propel themselves with many different types of movement. For example, flagella can exhibit a variety of structures and movement patterns. Bacterial flagella are helical and driven at their bases by a reversible rotary engine that rotates the attached flagellum to give a motion similar to that of a corkscrew (23). During runs, the filaments coalesce into a bundle that pushes the cell forward. By contrast, eukaryotic cells use flagella that resemble elastic rods and exhibit a beating motion; internally generated stresses give rise to a series of bends that propagate toward the tip. Spermatozoa realize locomotion by beating 
their flexible flagellum in a planar waveform $(24,25)$. In many bacteria, the direction of flagellar rotation is controlled by a chemotactic signal-transduction system that monitors environmental cues $(26,27)$. Elgeti et al. (28) reviewed the physics of locomotion of biological and synthetic microswimmers and the emergent collective behaviors of their assemblies, and Ozin et al. (29) and Sengupta et al. (30) addressed the propulsion of synthetic swimmers on the nanoscale and the development of nanomachines.

Another organelle that can help microorganisms swim is cilia, which look like short hairs covering the surface of the cell body. In mammals, many epithelial cells are ciliated in order to move material across the tissue surface. Cilia are used effectively in a wide variety of biological systems, from fluid transport to thrust generation. The mechanism of ciliary movement is based on the drag imbalance on a cylindrical element in combination with a nonreciprocal motion (31). In a low-Reynolds-number regime, the drag on a slender cylinder for motion perpendicular to its axis is approximately twice the drag when moving along its axis. When a slender cylinder is pulled at an oblique angle, it moves with an angle of attack owing to the drag imbalance. This results in a velocity vector that has components both in the pulling direction and perpendicular to this direction, which the organisms take advantage of when using flagella or cilia to generate motion.

Many prokaryotes move without using these rotary propellers to realize nonflagellar motility. Some marine cyanobacteria of the genus Synechococcus also swim without flagella. These abundant organisms actively swim at rates of 5-25 $\mu \mathrm{m} / \mathrm{s}$. A model to explain Synechococcus swimming has been proposed that involves the generation of traveling waves on the cell surface, perhaps as a result of oar-like movements of the cell surface spicules (32). Spiroplasma spp. locomotion is thought to be created by the contractile cytoskeleton, which functions as a linear motor. Differential length changes of the filament building blocks are thought to result in a change of the helical handedness of the cell and generate a moving kink (33). The process is then repeated, resulting in multiple kinks that move along the cell body. During this process, fluid is pushed from front to back, and the cells are propelled forward.

Motion principles and actuation mechanisms have been the subject of intensive investigation. The main challenges of developing micro- and nanorobots lies in their fabrication, powering, and steering. Inspired by the agility and plasticity seen in nature, efforts have recently focused on building flexible small-scale robotic devices. In this review, we summarize initial research 
efforts to produce micro- and nanorobots with flexible components and recent developments related to the incorporation of soft-matter architectures in small-scale robotic platforms.

\section{SPRING-MASS SYSTEMS}

A spring-mass system can store elastic potential energy when a spring is stretched or compressed by a mass. The elastic potential energy can be transferred into kinetic energy when the mass is released. A similar paradigm has been adapted for the development of microrobots. The microrobots can move in a stick-slip principle, which can be implemented by piezoelectric actuators, shape-memory alloys, and polymers.

Vollmers et al. (34) demonstrated a class of actuators utilizing wireless resonant magnetic actuation that accomplishes power and propulsion with a high degree of control. These firstgeneration microswimmers, known as MagMites, harvest magnetic energy from the environment and transform it to impact-driven mechanical force (Figure 1a). The robot structure, which has a largest dimension of $300 \mu \mathrm{m}$, consists of a conductive base frame carrying two small, asymmetric, soft magnetic masses. One mass (the body) stands on the substrate, while the other (the hammer) is supported by the spring and suspended above the substrate. Both the body and the hammer are made of a soft magnetic material. Because of magnetic shape anisotropy, the masses are magnetized in the direction of the easy (long) axis of the combined magnetic masses when exposed to an external magnetic field. This magnetization of the two masses generates a local magnetic gradient field and induces an attractive force between the two masses to pull them toward each other as well as a torque to rotate the microrobot to align its easy magnetic axis with the field (Figure 1b). The attractive force vanishes after the external magnetic field is removed, and the energy stored in the spring then pushes the two masses away. Generally, microrobots based on an arrangement of a relatively simple magnetomechanical spring-mass system operating close to resonance can absorb large amounts of energy from the driving signal. The actuation of this spring-mass system is based on harnessing the interactive forces between small magnetic bodies in a uniform magnetic field to drive the mechanism to resonance. This energy is then used to move the robot through its environment.

\section{<COMP: PLEASE INSERT FIGURE 1 HERE>}

Figure 1 (a) Scanning electron microscopy image of a MagMite. The robot measures $300 \mu \mathrm{m}$ 
square and is $70 \mu \mathrm{m}$ thick. Adapted from Reference 34 with permission from AIP Publishing. $(b)$ Interactive force of two soft-magnetic bodies when exposed to an external magnetic field. Depending on the device geometry, soft magnetic bodies in a uniform magnetic field can be made to either attract in a serial configuration (top) or repel in a parallel configuration (bottom). Maintaining a small distance between the bodies on a microrobot enables the generation of relatively large forces between them. (c) High-speed camera images that confirm the hammer motion. An increase of the field amplitude leads to the visually detectable higher swinging amplitudes and the eventual closing of the gap. The circle highlights where the deflection of the spring is mechanically limited by the attractor mass. Adapted from Reference 35 with permission from Sage Publications Inc. $(d)$ Scanning electron microscopy image of a PolyMite alongside polystyrene beads approximately $120 \mu \mathrm{m}$ in diameter. Adapted from Reference 36 with permission. Copyright (C) 2014 IEEE.

If a device were perfectly symmetrical in its oscillatory motion and actuated on a frictionless surface, it would vibrate in place without exhibiting any preferential direction of motion. Generating motion in a particular direction requires a rectification of the motion difference between the two directions of the stroke of the resonant body. This was achieved by driving the robots on an engineered substrate where the unidirectional force results from a phase-locked electrostatic clamping signal of approximately 30-80 V (35). The engineered substrate consists of interdigitated electrodes that induce a charge separation in the metallic robot frame and, thus, adjust the frictional forces. Aligning the on/off clamping phase with either forward or backward strokes of the oscillation can drive the robot forward or backward. Placing the device in an oscillating external magnetic field whose frequency is near the resonant frequency of the device can generate a net displacement in dry or wet environments (35). The hammer motion has been verified by high-speed optical camera imaging (Figure 1c). The MagMite can move controllably at high speeds when driven in air $(12.5 \mathrm{~mm} / \mathrm{s}$ or 42 body lengths per second), and the force that it generates is strong enough to push micrometer-sized objects, which shows its potential applications for sorting and extracting delicate biological and biochemical samples. The same group developed a second generation of wireless resonant magnetic microrobots known as PolyMites (Figure 1d) by reducing the time, cost, and the complexity of the MagMite fabrication process. Two symmetric polymer springs are used to connect the two masses, and thus the movement of the device is more stable in the lateral direction. The masses are present on the same plane as the springs to ensure that the magnetic attraction forces and the restoration forces of the spring are in the same plane. The use of a polymer enables the design of a stable spring system without a significant increase in stiffness. Tung et al. (36) demonstrated a speed of over 
$20 \mathrm{~mm} / \mathrm{s}$ (40 body lengths per second) when the PolyMite was driven in air with an applied magnetic flux density of $4 \mathrm{mT}$ with a reduced drift effect.

\section{MICROMACHINES WITH ACTUATED ARMS, HINGES, AND CILIA}

Flexures and hinges can significantly increase the dexterity when performing complex manipulation tasks, such as gripping and clamping. These novel modes of actuation can enhance the capabilities of microrobots for transport and assembly of microobjects in 3-D space. Microgrippers are tiny mechanical devices that can be actuated to close their arms and generate a confinement. Conventional microgrippers are tethered to electric cables or pneumatic tubes, and these physical connections significantly limit their maneuverability.

Gracias and coworkers $(37,38)$ developed star-liked microgrippers that are chemically triggered to open and close by acetic acid and hydrogen peroxide, respectively. The grippers open and close based on chemical etching or enzymatic degradation, which results in mechanical property changes within trilayer joints patterned within the gripper, and no external power is needed for operation. The actuation of the gripper using a multilayer thin film demonstrated the utility of the gripper by picking and placing $200-\mu \mathrm{m}$-diameter tubes and beads $(37,38)$. A drawback of the approach is that the chemicals used to trigger microgrippers are not compatible with living organisms. In another work from the same group, Leong et al. (39) fabricated a similar design using a polymer and a stressed bimetallic thin film patterned between rigid phalanges and developed microgrippers that are free of constraining tubes or wires (Figure 2a). Patterned by photolithography with metallic-polymer joints, their microgrippers resemble submillimeter-sized crab pincers and use a design inspired by the joints of arthropods. The gripping action arises from a prestressed bimetallic layer that is prevented from flexing by a segment of organic polymer at each joint. The tension can be released by increasing the temperature above $40^{\circ} \mathrm{C}$, introducing the microgripper into a caustic solution, or exposing the microgripper to a variety of common biological reagents, such as L-glutamine. The grippers can be actuated en masse, even while spatially separated. They are used to perform diverse functions, such as picking up a bead on a substrate or retrieving cells from a bovine bladder tissue. A similar device is used to excise tissue samples from real organs and hard-to-reach places within a live animal. By performing the device in the gastroenterological procedures of ex vivo tissue excision of a porcine liver and in vivo tissue retrieval from a porcine biliary tree, both intact cells 
and high-quality RNA and DNA from the retrieved tissue can be extracted, which can then form the basis for cytologic and molecular biology analyses to establish diagnoses of cancer, inflammatory conditions, or other conditions $(40,41)$. The same group demonstrated the capture of live mouse fibroblast cells and red blood cells with their untethered grippers (42). To approach remotely targeted tissue and monitor microgripper performance, Yim et al. (43) used a magnetic capsule endoscope to carry, release, and retrieve thermosensitive untethered microgrippers.

\section{<COMP: PLEASE INSERT FIGURE 2 HERE>}

Figure $2(a)$ Thermally triggered actuation, magnetic manipulation, and bead capture of a microgripper. Adapted from Reference 39. Copyright (c) 2009 National Academy of Sciences. $(b)$ Two types of remotely actuated untethered magnetic microgripper. (Left) Torque-based addressable microgripper. (Right) Force-based addressable microgripper. Adapted from Reference 44 with permission. Copyright (C) 2014 Wiley. (c) Schematic drawing of Purcell's scallop with reciprocal motion and 3-D model of scallop swimmers with different dimensions. Adapted from Reference 45 with permission from Nature Publishing Group. $(d)$ Programming of soft composite materials into various shapes. (Upper left) A reversible CMU logo. (Upper right) A jellyfish-like robot equipped with two soft tentacles. (Bottom left) A spermatozoid-like undulating soft swimmer. (Bottom middle and right) The recovery stroke motion and power stroke motion of a beating artificial cilium, respectively. Adapted from Reference 50. Copyright (C) 2016 National Academy of Sciences.

Mobile microgrippers can also be remotely actuated by external magnetic fields. For example, micromagnets are mounted at the end of each flexible arm, and the gripper is closed by the application of a uniform magnetic field (44) (Figure 2b). Another magnet attached on the body provides locomotion for precise transportation, orientation, and programmable threedimensional assembly of microparts. In addition to their gripping capabilities, actuated hinges and bending filaments enable the construction of propellers. For example, the microscallop developed by Fischer and coworkers (45) is a single-hinge symmetric microswimmer that can propel itself in complex fluids by reciprocal motion. It consists of two thick shells connected by a thin, narrow hinge (Figure 2c). Micromagnets are attached to each shell so that when they are exposed to an external magnetic field, the two magnets reorient and align with the field and thus close the shells of the microscallop. Dieny and coworkers (46) employed a similar design to fabricate nanotweezers consisting of two magnetic microelements bound by a flexible gold nanohinge. The nanotweezers switched from an open to a closed conformation triggered by an external magnetic field. 
Choi and coworkers (47) recently developed ciliary microrobots that exhibit nonreciprocal magnetic actuation. These devices were manufactured using two-photon lithography followed by sputtering of a nickel-titanium bilayer. Applying an external stepping magnetic field caused the cilia to adopt different stroke patterns, thereby propelling the microrobotic platform.

An interesting approach for the fabrication of microgrippers is the use of magnetic polymer composites. Magnetic particles can be used not only to provide magnetic properties to the polymer matrices but also to predefine the orientation of the components of the device if conveniently aligned or premagnetized. A pioneering effort in magnetically programmed polymer composite devices was realized by Kwon and coworkers (48), who reported a microactuator with programmed magnetic anisotropy. The proposed method enables each component of the microstructure to actuate in predefined directions by aligning superparamagnetic particles during the fabrication sequence. In other words, the easy magnetization axis of each component is predefined, so that complex movements, such as a snake-like mechanism, can be achieved. Because these kinds of systems use superparamagnetic nanoparticles, they do not retain magnetization in the absence of an applied magnetic field. Following a similar approach, Zhang \& Diller (49) employed elastomeric magnetic composites containing hard magnetic microparticles to create tetherless programmable mobile micrograspers. These devices consist of four limbs and are $3.5 \mathrm{~mm}$ in length from tip to tip in the open configuration. The structure can shrink down to a 700- $\mu \mathrm{m}$ cube in the closed state. To program their magnetic behavior, the magnetic microparticles are premagnetized depending on their location in the microgripper. The authors demonstrated grasping and transportation of microobjects using both magnetic field gradients and rotating magnetic fields. Sitti and coworkers (50) introduced a universal programming methodology to generate the required magnetization profile and actuating fields for magnetic soft elastomeric composite materials in order to achieve new time-varying shapes by analyzing the deformation kinematics. Thus, versatile shapes, such as a reversible CMU logo, a jellyfish-like robot, a spermatozoid-like undulating swimmer, and an artificial cilium, can be transformed from a soft beam (Figure $2 d$ ).

\section{SEGMENTED NANOSTRUCTURES WITH FLEXIBLE JOINTS}

There are few examples of nanorobots with flexible joints or hinges, primarily because the implementation of flexible nanoscale building blocks is challenging. The first example of a 
swimmer containing very small links was developed by Dreyfus et al. (51). These devices consist of a linear chain of superparamagnetic microbeads connected with DNA strands (Figure $3 a$ ). A red blood cell is fixed to the end of the chain in order to break the symmetry when the swimmer is actuated and thereby achieve nonreciprocal motion. Snezhko \& Aranson (18) also showed that colloidal suspensions of nickel spherical microparticles self-assemble as magnetic asters and arrays of asters in magnetic colloidal suspensions at the interface between two immiscible liquids. The asters and aster arrays can be manipulated using small external alternating magnetic fields and are able to execute several microrobotic tasks, such as capturing, transporting, and positioning.

\section{<COMP: PLEASE INSERT FIGURE 3 HERE>}

Figure 3 (a) Schematic representation of a flexible magnetic filament. The magnetic particles are coated with streptavidin (red crosses). Under an applied magnetic field, the particles form filaments. Double-stranded DNA with biotin at each end can bind the particles together via the specific biotin-streptavidin interaction. Adapted by permission from Macmillan Publishers Ltd: Nature (51 copyright (C) 2005. (b) Schematic of two-arm nanoswimmers. Applying an oscillating magnetic field leads to freestyle swimming of the nanorobot, with two nanoarms wobbling alternatively to generate actuation. Adapted from Reference 56 with permission. Copyright (C) 2017 American Chemical Society. (c) Planar undulations of composite multilink nanowire-based chains (diameter $200 \mathrm{~nm}$ ) induced by a planar-oscillating magnetic field. Those chains comprise an elastic eukaryote-like polypyrrole tail and rigid magnetic nickel links connected by flexible polymer bilayer hinges. Adapted from Reference 58 with permission. Copyright (C) 2015 American Chemical Society. (d) Structural configuration and fabrication of tethered micromotors with an open angle, connected in the orders of $\mathrm{Pt} / \mathrm{Au}-\mathrm{Au} / \mathrm{Pt}($ left $)$ and $\mathrm{Pt} / \mathrm{Au}-\mathrm{Pt} / \mathrm{Au}$ (right). Abbreviations: AE, [Auxiliary electrode]; EDC, [1-ethyl-3-(3(dimethylamino)propyl)carbodiimide hydrochloride]; PAA, poly(acrylic acid); PAH, poly(allylamine hydrochloride); RE, [Reference electrode]; WE, [Working electrode. Adapted from Reference 62 with permission. Copyright (C) 2017 American Chemical Society.

Swimmers with flexible parts built entirely of synthetic nanostructures were later manufactured by Wang and coworkers (52-54) using template-assisted electrodeposition. These swimmers consist of nanowires containing two or three segments of different metals, with one segment exhibiting flexibility. The chiral deformation along the flexible segment is responsible for the propulsion of these devices. The flexible segment is made of silver partially etched using hydrogen peroxide. Another segment is made of nickel, which enables the motion of these swimmers by means of rotating magnetic fields. Nickel-silver swimmers exhibit a speed of 21 $\mu \mathrm{m} / \mathrm{s}$ at an actuation frequency of $35 \mathrm{~Hz}$. These speed values are close to those reported for microorganisms such as Escherichia coli, which are on the order of $30 \mu \mathrm{m} / \mathrm{s}$. In vitro delivery of 
drug-loaded beads to HeLa cancer cells in biological media was demonstrated using nickelsilver nanoswimmers. Trisegmented nickel-silver-gold nanowires were also developed. The third segment, which is made of gold, is incorporated to tune the forward or backward motion of the swimmer. Wang's group further adapted this strategy to fabricate nanofish, which consist of two gold segments that act as a head and a caudal fin, respectively, and two magnetic body segments made of nickel. These parts were linked with three flexible, porous silver joints (55). The group employed the same manufacturing approach to create a symmetric multilinked magnetic two-arm nanomachine capable of efficient freestyle swimming (56) (Figure 3b). Synchronized oscillatory deformations account for the observed freestyle locomotion mechanism of the nanoswimmers.

Another type of flexible microrobot that mimics sperm flagellar motion has also been engineered (57). This microrobot consists of a structure resembling a sperm cell with a magnetic head and a flexible tail $42 \mu \mathrm{m}$ and $280 \mu \mathrm{m}$ in length, respectively. The magnetic head includes a 200-nm-thick cobalt-nickel layer. This layer provides a dipole moment and allows the flexible structure to align along oscillating weak (less than $5 \mathrm{mT}$ ) magnetic field lines, and hence generates a propulsion thrust force that overcomes the drag force. When driven with alternating weak magnetic fields of $45 \mathrm{~Hz}$, the swimmer can reach an average speed of $158 \mu \mathrm{m} / \mathrm{s}$.

Nelson and coworkers (58) recently reported for the first time the realization of Purcell's three-link nanoswimmers using a fabrication strategy described by Ozin and coworkers (59). In his 1977 paper "Life at Low Reynolds Number" (60), the Nobel laureate Edward Purcell presented the scallop theorem, which states that propulsion at micro- and nanoscales is possible only if a swimmer can break time-reversal symmetry. His simplest model consists of a swimmer containing three links connected by two rotary joints. This design can generate a traveling wave and thus achieve nonreciprocal net motion. The hinged nanoswimmers reported by Nelson and coworkers (58) exhibit an overall length of $15.5 \mu \mathrm{m}$ and comprise an elastic eukaryote-like polymer tail made of polypyrrole and rigid magnetic nickel links (200 nm in diameter) joined by soft, flexible polymeric hinges (Figure $3 c$ ). To form the flexible hinges, segmented nanowires containing sacrificial gold layers were coated with bilayered films of cationic poly(allylamine hydrochloride) (PAH) and anionic polystyrenesulfonate (PSS) by means of layer-by-layer deposition. Etching the gold segments forms tubular polymeric joints. The nanoswimmers are magnetically actuated by planar oscillating external fields in a $65 \%$ glycerol solution (the 
dynamic viscosity of which is around $15 \mathrm{mPa} \cdot \mathrm{s}$ at $20^{\circ} \mathrm{C}$ ) and display a nonreciprocating motion. The work by Nelson and coworkers (58) also includes a comparison of the motion dynamics and swimming performance of one-, two-, and three-link nanoswimmers. All swimmers show undulatory locomotion and an optimal frequency that maximizes speed. Interestingly, the swimming behavior of one- and two-link swimmers resembles the motion of Purcell's flexible oar, while the three-link Purcell-like swimmer exhibits an S-like motion with the appearance of a traveling wave under planar oscillatory magnetic fields. The analyzed maximum speeds of one-, two-, and three-link swimmers are $0.43,0.70$, and 0.93 body lengths per second, respectively. The same group used the one-link configuration and managed to propel the swimmers by both traveling and standing acoustic waves (61). The propulsive force in these nanorobots is still under investigation, but it is believed to be due to the vibration of the swimmer and the stress developed due to localized vortices. For propulsion under traveling waves, the use of a soft, flexible tail is crucial. The swimmers were able to navigate in straight lines through water containing stationary polystyrene particles.

Capitalizing on the fabrication strategy of Ozin and coworkers (59), Yoshizumi \& Suzuki (62) realized self-propelled catalytic nanomachines consisting of bisegmented gold-platinum nanorods connected with bended polymeric joints. Figure $3 d$ shows two different configurations and the fabrication sequence of these structures. In this case, the joints are formed by etching sacrificial segments of silver. Interestingly, poly(acrylic acid) (PAA) is employed as the anionic film in the layer-by-layer coating of the structures. To create bendable joints, the structures were partially embedded in paraffin so that one of their longitudinal sides was exposed to an alkaline solution. This treatment causes the partial cleavage of the cross-linking bonds by hydrolysis and leads to an increase in the swelling properties of the polymeric layer, which in turn causes the bending at the joint. The flexibility of the joints can be tuned as a function of the number of bilayers in the polymeric coating. When the structures are immersed in a solution of hydrogen peroxide, nanoswimmers consisting of $\mathrm{Pt} / \mathrm{Au}-\mathrm{Au} / \mathrm{Pt}$ with a soft joint exhibit bending motion; by contrast, $\mathrm{Pt} / \mathrm{Au}-\mathrm{Pt} / \mathrm{Au}$ nanoswimmers with a rigid bent joint show rotation.

\section{SOFT-BODIED MICROROBOTS}

Smart materials can change their physical or chemical properties when exposed to an external stimulus, such as heat, mechanical stress, concentration of certain chemical species, light, 
ultrasound, or a magnetic or electric field (63-65). Materials that fall into this category include but are not limited to shape-memory alloys and polymers (66), magnetostrictive and piezoelectric compounds $(67,68)$, stimulus-responsive hydrogels $(69,70)$, and chromogenic materials (71). Among these materials, stimulus-responsive hydrogels are promising candidates for soft micro- and nanorobots (72-76). These hydrogels can be sculpted into deformable structures that can change their shape and volume when subjected to environmental stimuli, such as a change in $\mathrm{pH}$, a change in temperature, or a chemical concentration gradient (77-80). Because of these features, hydrogels have been employed to fabricate structures for encapsulation and controlled release of drugs and cells and have led to new paradigms in the development of adaptive and actuating materials and structures for minimally invasive surgery $(14,15,66,81-83)$.

A simple and attractive method to fabricate smart hydrogel-based 3-D architectures is exploiting controlled self-folding behavior (84). When two or more materials that swell or contract differently in response to a particular chemical or physical change are coupled together, the composite structure undergoes deformations that lead to folding or buckling. This origamilike fabrication process has been used to engineer multifunctional soft microrobots that are remotely controlled using magnetic and optical manipulation systems. Of all the hydrogels, material compositions based on poly( $N$-isopropylacrylamide) (pNIPAM) are preferred because they can be photopatterned to create a wide range of morphologies at physiological temperatures (85-88). Hydrogel actuators can be created using bigel strips composed of partially interpenetrated hydrogel networks with different swelling properties. Hu et al. (89) demonstrated this concept using bilayers of pNIPAM and acrylamide that had drastically different swelling behaviors in response to different temperatures or solvent compositions. Nelson and coworkers (90) developed hydrogel bilayers with jellyfish-like shapes to provide a protected compartment that can be rapidly opened by brief near-infrared light exposure or by increasing the environmental temperature to over $40^{\circ} \mathrm{C}$ (Figure $4 a$ ). With this concept of compartmentalization, the dose of the therapy can be increased while protecting the payload during the transport. The micromachines can change their shapes by folding and unfolding upon thermal stimulation. In their closed form, spontaneous drug diffusion from tubular structures is limited, and the drag forces are lower compared with those of their unfolded state. Performing transport in the folded state and switching to an unfolded state at the target location can improve 
both maneuverability and the specificity of the therapy. Park and coworkers (65) developed a hybrid actuated soft microgripper with the ability of locomotion. The gripper was made of poly(2-hydroxyethyl methacrylate) (PHEMA) and poly(ethylene glycol) diacrylate (PEGDA) with iron(II,III) oxide particles $\left(\mathrm{Fe}_{3} \mathrm{O}_{4}\right)$. PHEMA is a $\mathrm{pH}$-responsive gel responsible for the trapping and unfolding motion of the soft microgripper in $\mathrm{pH}$-varying solution, and the PEGDAwith- $\mathrm{Fe}_{3} \mathrm{O}_{4}$ layer is employed for the locomotion of the soft microgripper in the magnetic field.

\section{<COMP: PLEASE INSERT FIGURE 4 HERE>}

Figure 4 (a) A microrobotic platform combining the advantages of self-folding near-infraredsensitive polymer bilayers, magnetic alginate microbeads, and a 3-D manipulation system for targeted, on-demand drug and cell delivery. Adapted from Reference 90 with permission. Copyright (C) 2014 Wiley. (b) Formation of compound micromachines with a bilayer head and monolayer tail. The helical angle is regulated by the alignment angle of magnetic nanoparticles. Adapted from Reference 96 with permission from Nature Publishing Group. (c) Deformation of an anchored cylindrical microrobot under a periodic light pattern traveling from left to right. The green overlay shows the illuminated area, the yellow dotted line shows the rest configuration, and the red dashed line shows the deformed profile. Adapted from Reference 104 with permission from Macmillan Publishers Ltd: Nature Materials, copyright (c) 2016.

Mechanical motion derived from the large and reversible swelling response of hydrogel in varying thermal or $\mathrm{pH}$ environments shows a capacity for large strain. However, owing to the low modulus of hydrogels, they have limited gripping ability to complete robotic tasks such as pick and place. Therefore, hinged microgrippers with high-modulus layers have been fabricated (91). A biodegradable, photopatternable poly(propylene fumarate) (PPF) as a rigid panel and biocompatible poly( $N$-isopropylacrylamide-coacrylic acid) (pNIPAM-AAc) as a flexible hinge have been used to build theragrippers (92). Because of the porosity in the grippers, fluorescent dyes and commercial drugs such as mesalamine and doxorubicin could be loaded. The drugs eluted from the grippers for up to seven days with first-order release kinetics. In an in vitro model, theragrippers enhanced delivery of doxorubicin as compared with a control patch (87). Folding in nature can exhibit complex behaviors, which strongly depend on the geometry and swelling paths that may result in multistep folding. Ionov and coworkers (93) demonstrated that the shape of isotropic polymer bilayers is able to direct folding in a sophisticated manner. Hydrogel films can undergo sequential folding steps by forming various 3-D shapes with sharp hinges. Bilayer polymer films placed on a substrate start to fold from their periphery, and the number of wrinkles or tubes formed decreases until the angle between adjacent wrinkles or tubes 
approaches $130^{\circ}$. After the wrinkles along the perimeter of the film form tubes, further folding proceeds along the lines connecting the vertexes of the folded film. The folding goes along the lines that are closer to the periphery of the films. Folding of the rays may result in blocking of the neighboring rays if the angle between the base of the folded ray and the shoulders of the neighboring rays approaches $180^{\circ}$.

Soft responsive hydrogels can be used to program both bending and swelling, thereby providing access to a wide array of complex 3-D forms. As an alternative method, a single-layer hydrogel sheet has been programmed with chemically distinct, fiber-like regions that exhibit differential shrinkage and elastic moduli under the application of external stimulus $(94,95)$. The hydrogel sheet can realize a planar-to-helical 3-D shape transformation. This programming approach can be generalized by incorporating reinforcing magnetic nanoparticles inside soft hydrogel layers. Nelson and coworkers (96) developed various bio-inspired self-folding micromachines with complex body plans, reconfigurable shapes, and controllable motility by incorporating magnetic nanoparticles into the porous hydrogel body (Figure $4 b$ ). Selective reprogramming of the mechanical design and magnetic anisotropy can modulate the swimming characteristics of the micromachines. The authors fabricated magnetic bilayers that fold into helical shapes, which consist of a nonswelling supporting hydrogel layer composed of PEGDA selectively patterned on a swelling thermoresponsive pNIPAM hydrogel layer. The motility of these devices is induced through the addition of magnetic nanoparticles in the hydrogel layers and tailored by magnetically aligning the particles during polymerization. Selective alignment of magnetic nanoparticles in different compartments results in a multitude of magnetization axes. Magnetic nanoparticles are also utilized as reinforcing components, and the folding axis of each compartment can be programmed through selective alignment of magnetic nanoparticles. Consequently, self-folding transforms a two-dimensional hydrogel microstructure into a 3-D biomimetic microswimmer with a tailored shape and magnetic anisotropy. While the helices can corkscrew with rotating magnetic fields, the structures in their unfolded state only rotate in place. The role of shape shifting in locomotion can be amplified by linking propulsion to the shape of the microrobot. A shape transformation can be initiated to stop motion at the target location by engineering helical robots that unfold because of the unique properties of the microenvironment at the target location (i.e., a change in $\mathrm{pH}$ ). After folding, the propeller geometry of flagellated 
soft microrobots has a great effect on their locomotion. The motility of flagellated microrobots can be greatly influenced by curved, helical, and straight tails (97).

A typical approach to autonomously power micro- and nanoscale tools involves the catalytic conversion of chemical energy (energy-rich molecules in solution) into mechanical energy. Swimmers incorporate a catalyst as a part of their structure for the decomposition of a chemical fuel. Generally, $\mathrm{H}_{2} \mathrm{O}_{2}$ or glucose are used as the fuel, resulting in an autonomously propelled swimmer that does not require external guidance or power (98-102). The folding and unfolding mechanism of stimulus-responsive hydrogel sheets can also be used to regulate chemical reaction rates. Schmidt and coworkers (103) reported a coating of thin platinum film on flexible thermoresponsive polymeric layers. The microswimmer can reversibly fold and unfold by applying changes in temperature to the hydrogen peroxide solution in which they are immersed, which in turn influences the dynamics of catalytic reaction, thus controlling the swimming speed of this flexible, bubble-propelled micromotor.

Micro- and nanoswimmers based on thermo- or $\mathrm{pH}$-responsive hydrogels are difficult to miniaturize, as the maintenance of temperature or $\mathrm{pH}$ differences becomes difficult at the smaller scale, which makes the swimmer ineffective. In addition, large temperature or $\mathrm{pH}$ differences are not compatible with most biological systems. In a recent study, Palagi et al. (104) used liquidcrystal elastomers with photoisomerizable azobenzene groups to fabricate a self-propelled biomimetic microrobot, employing structured light to power and control intrabody shape changes (Figure 4c). Liquid-crystal elastomers can exhibit large shape changes under light stimuli (105). The development of laser lithography and molecular alignment engineering has enabled controlled illumination of these soft materials in arbitrary 3-D geometries with a predefined actuation performance. The directed motion was generated by periodically deforming their soft bodies. The devices can be fully controlled externally. The light-induced deformation varies with illumination conditions, allowing one monolithic liquid-crystal elastomer structure to perform different actions with high spatial and temporal resolution. When such structures are combined with other types of physical fields, intrinsically more functional microrobots that demonstrate more complex behaviors can be expected (106). Apart from these elastomers, DNA-cross-linked polyacrylamide hydrogels have also been investigated as a way to produce addressable swelling throughout bulk hydrogel $(107,108)$. A recent study reported that DNA molecules can induce a 100-fold volumetric hydrogel expansion by successive extension of cross-links using a DNA 
hybridization cascade where DNA sequences consist of hydrogel cross-links and corresponding hairpins (109). The authors developed a delicate photolithography process to pattern DNA hydrogels into precisely defined architectures, thus allowing the fabrication of well-defined DNA hydrogel shapes capable of arbitrary shape change in 3-D.

Supramolecular structures such as polymersomes and other assemblies of polymers have also been used to create soft-bodied small-scale swimmers. Wilson et al. (110) created polymersomebased nanoswimmers that can be propelled by the catalytic decomposition of hydrogen peroxide. The polymersomes, also known as stomatocytes, are bowl-shaped ensembles of amphiphilic block copolymers [poly(ethylene glycol)-b-polystyrene]. The opening, or mouth, of these structures allows for entrapping catalytically active nanoparticles in their inner cavities. The decomposition of hydrogen peroxide inside the stomatocytes produces oxygen bubbles, which are responsible for the propulsion of the swimmers. Wilson and coworkers (111) also demonstrated the chemotactic behavior of stomatocytes toward higher concentrations of hydrogen peroxide in a microfluidic system, also using hydrogen peroxide-excreting neutrophils. By capitalizing on the diamagnetic properties of block copolymers, the same group recently demonstrated the actuation of the stomatocytes' mouths using strong magnetic fields (112). The backbone of the amphiphiles tends to align perpendicularly to an applied magnetic field owing to their negative magnetic susceptibility. Consequently, a strong magnetic field triggers the stomatocytes' shapes to switch from a spherical to a prolate morphology. The authors demonstrated that it is possible to use this strategy to capture and release nanoparticles. The group has also engineered biodegradable stomatocytes by introducing a disulfide moiety between the polymers forming the amphiphile (113). In the presence of glutathione, an intracellular reducing agent present in cells, the disulfide bond is cleaved, causing the stomatocytes to aggregate and release drugs.

Bodies of small-scale machines have also been fabricated by means of layer-by-layer deposition of bilayers consisting of polymers, hydrogels, and proteins. For example, He and coworkers (114) reported on self-assembled tubular nanorockets made of polymer multilayers of chitosan and alginate. The nanoswimmers were fabricated by template-assisted layer-by-layer deposition using polycarbonate nanoporous membranes, and platinum nanoparticles were deposited in the inner cavity of the tubular nanostructures to allow for the catalytic propulsion of the nanorockets using hydrogen peroxide. The same group used a similar fabrication approach to 
create polymer multilayer nanorockets made of layer-by-layer deposited bilayers of poly(allylamine hydrochloride) and poly(styrenesulfonic acid) (115). The structures were functionalized with platinum nanoparticles and a thin gold layer, the latter of which serves to propel the structure under near-infrared illumination without the need for hydrogen peroxide. The propulsion is attributable to thermophoresis. He and coworkers (116) have also manufactured biodegradable protein-based nanorockets made of bilayers of bovine serum albumin/poly-L-lysine. These nanorockets comprise a thermoresponsive gelatin hydrogel matrix containing gold nanoparticles, doxorubicin, and catalase. The rockets are propelled by biocatalytic bubble propulsion using hydrogen peroxide, which can diffuse into a gelatin matrix and is readily decomposed by catalase. Magnetic nanoparticles are electrostatically assembled around the rockets, allowing for their magnetic guidance. Release of doxorubicin is achieved by exposing the rockets to near-infrared illumination. The heat generated by gold nanoparticles triggers the gel-sol phase transformation of gelatin, which in turn enables the drug to diffuse out of the nanorockets.

\section{BIOHYBRID ACTUATION}

Biohybrid microswimmers have been introduced as an alternative strategy for building untethered biological micromachines. A variety of microorganisms (flagellated bacteria) and contractile mammalian cells (cardiac muscle and skeletal muscle) have been utilized as microengines and integrated with functional synthetic materials, producing bending or deformation of the functional materials to initiate motion (117-123). These biological machines showed superior abilities for sensing, processing, and responding to environmental signals in real time. They are fueled by the surrounding biological fluid and offer the opportunity to efficiently convert chemical energy into mechanical work for 3-D propulsion. The development of gene technology and tissue engineering has made it feasible to design and fabricate complex biological machines by incorporating multiple cell types, such as neurons for signal processing or endothelial cells for vascularization and nutrient transport. For a detailed review of biohybrid microsystems, we refer the reader to References 124 and 125; here, we focus on the generation of motion from compliant mechanisms.

Cardiomyocytes can contract rhythmically by the activation of actin-myosin, and the process is coordinated in space and time through excitation-contraction coupling. Soft robotic 
microdevices that can crawl on planar surfaces or swim in fluidic environments have been engineered by integrating cardiomyocytes with elastic substrates. While the cells provide either spontaneous or paced contractile function, the synthetic component allows the fabrication of machines with functional forms. One solution for building such a device is culturing cardiac muscle tissue on polymer thin films. The constructs, termed muscular thin films, were designed to perform biomimetic tasks by varying tissue architecture and thin-film shape (126). The cardiac muscle can power rudimentary walking, swimming, and gripping in vitro. In a similar design, cardiomyocytes were cultured onto grooved polydimethylsiloxane (PDMS) surfaces that allow for high-density patterning of cells onto the synthetic scaffold. The micromachine continued to function over a period of 10 days and showed sustained long-term functionality (127). Compared with a flat beam, the grooved surface resulted in an increase in force (88\%) and bending (40\%) with an average walking speed of $140 \mu \mathrm{m} / \mathrm{s}$.

Inspired by the architecture and propulsion mechanism of the jellyfish, a bioengineered muscular thin film performed controlled swimming in a medium bath once the contraction of the muscle tissue was synchronized with an externally generated electric field (128) (Figure 5a). As a further advancement, Park et al. (129) built a 1/10th-scale version of a ray fish with a microfabricated gold skeleton and a rubber body powered by rat heart muscle cells. The cardiomyocytes were genetically engineered to respond to light cues (Figure $5 b$ ), so that the undulatory movements propelling the robot through water followed a light source. In addition to swimming, these biohybrid constructs can move on surfaces. Chan et al. (130) seeded cardiac cells on stereolithographically printed hydrogel microcantilevers; by harnessing an asymmetric design and the synchronous contraction of the cardiac cell sheet, they demonstrated a walking motion of the machine. The locomotion was predicated on varying the friction between the robot legs and the substrate during the course of contraction. Saif and coworkers (131) developed a self-propelled biohybrid swimmer that consisted of an elastic filament with a rigid head and a slender tail on which cardiomyocytes were selectively cultured (Figure $5 c$ ). The cardiomyocytes contracted and deformed the filament to propel the swimmer at 5-10 $\mu \mathrm{m} / \mathrm{s}$, consistent with model predictions. The authors also demonstrated a two-tailed swimmer moving at higher speeds.

\section{<COMP: PLEASE INSERT FIGURE 5 HERE>}

Figure 5 (a) Body design of a jellyfish (left) and a free-swimming medusoid construct (right). 
Adapted from Reference 128 with permission from Macmillan Publishers Ltd: Nature Biotechnology, copyright 2012. (b) Bioinspired design of a tissue-engineered ray. As shown on the left, the architecture consists of four layers. Upon optical stimulation, the tissue-engineered ray induces sequential muscle activation via serpentine-patterned muscle tissues, generates undulatory locomotion, and sustains steady forward swimming. It changes direction by generating asymmetric undulating motion between the left and right fins, modulated by a light pulse. Adapted from Reference 129 with permission from AAAS. (c) Concept design of a biohybrid flagellum. Spermatozoa swim at a low Reynolds number by generating complex, timeirreversible waveforms throughout a long, slender tail (left). A proposed biohybrid swimmer (middle) consists of an elastic filament with a rigid head, a compliant tail, and a single small cluster of contractile cells. On the right is a scanning electron microscopy image of a fabricated polydimethylsiloxane (PDMS) filament near the head-tail junction. Adapted from Reference 131 with permission from Nature Publishing Group. (d) Single-leg stimulation of a geometrically symmetric structure that drives locomotion in the direction of the stimulated leg predicted via finite element analysis and confirmed via optical stimulation. Adapted from Reference 133. Copyright @ 2016 National Academy of Sciences.

Skeletal muscle is the primary generator of actuation in animals, acting as a contractile power source. In vivo, skeletal muscle exhibits organized modular tissue architecture on a range of length scales and supports uniaxial force production. Unlike the significant spontaneous contractility exhibited by cardiac tissue, skeletal muscle has several features that make it an appealing actuator for the production and regulation of force for locomotion. Skeletal muscle couples contractile ability and strong force generation with high controllability by means of external stimulation.

Sakar et al. (132) showed that the variability in arrangement combined with spatiotemporal control of activity provides controlled, graded tension along with multi-degree-of-freedom actuation. Skeletal muscle cells were self-assembled into 3-D muscle strips capable of sufficient force generation to power net locomotion of biological machines. When coupled to a 3-D-printed flexible skeleton, these actuators drove directional locomotion (1.3 body lengths per minute) and 2-D rotational steering in a precisely targeted and controllable manner. The motion of these devices can be controlled by electrical and optogenetic stimulation $(133,134)$. The muscle actuators dynamically adapt to their surroundings by adjusting performance in response to training stimuli (133) (Figure $\mathbf{5} \boldsymbol{d}$ ). This adaptive behavior that reacts to external triggers enables a variety of applications that require dynamic sensing and actuation.

\section{CONCLUSIONS}


We have summarized several types of deformable micro- and nanomachines in terms of design, fabrication, and control principles, with a focus on spring-mass systems, devices with actuated arms and hinges, segmented nanostructures with flexible joints, soft-bodied microrobots, and biohybrid actuation. The advent of new micro- and nanotechnologies makes it possible to design and fabricate different types of soft micro- and nanorobots. Since the introduction of the first artificial microscopic swimmers, many prototypes have been developed for a number of biomedical applications, ranging from in vitro single-cell surgery to in vivo targeted drug delivery. Fabrication of wireless microrobots is fundamentally limited by scaling issues, and power and control are highly coupled. As a result, most actuation schemes are based on translation and rotation of rigid components with homogeneous material compositions and simple mechanical designs. Achieving complex dexterous motion with precise shape control will require novel energy-transduction mechanisms and material systems. This emerging bioinspired field holds great promise for augmenting the capabilities of traditional hard microrobots with polymeric modules that mimic biological actuators. The excitement generated by soft and flexible micro- and nanorobots centers on their simple design, low weight and cost, and adaptability at lower temperatures and in aqueous environments as well as the possibility of mimicking human functionality through their robotic functions. The future of soft micro- and nanorobots must move toward higher degrees of robustness, efficiency, and biocompatibility for the biological applications intended. Other future possibilities for these micromachines could include self-detection, self-diagnosis, self-repair, and the ability to grow, self-replicate, and display autonomous behavior. The development of soft micro- and nanorobots will continue to progress alongside other fields, including materials science, robotics and automation, and nanotechnology.

\section{DISCLOSURE STATEMENT}

The authors are not aware of any affiliations, memberships, funding, or financial holdings that might be perceived as affecting the objectivity of this review.

\section{ACKNOWLEDGMENTS}


We would like to acknowledge European Research Council Advanced Grant 743217 - Soft

Micro Robotics (SOMBOT) and Swiss National Science Foundation grant 200021_165564.

\section{LITERATURE CITED}

1. Nelson BJ, Kaliakatsos IK, Abbott JJ. 2010. Microrobots for minimally invasive medicine. Annu. Rev. Biomed. Eng. 12:55-85

2. Jager EWH. 2000. Microrobots for micrometer-size objects in aqueous media: potential tools for single-cell manipulation. Science 288:2335-38

3. Ahmed D, Dillinger C, Hong A, Nelson BJ. 2017. Artificial acousto-magnetic soft microswimmers. Adv. Mater. Technol. 2:1700050

4. Hu C, Aeschlimann F, Chatzipirpiridis G, Pokki J, Chen X, et al. 2017. Spatiotemporally controlled electrodeposition of magnetically driven micromachines based on the inverse opal architecture. Electrochem. Commun. 81:97-101

5. Rogoz M, Zeng H, Xuan C, Wiersma DS, Wasylczyk P. 2016. Light-driven soft robot mimics caterpillar locomotion in natural scale. Adv. Opt. Mater. 4:1689-94

6. Huang TY, Sakar MS, Mao A, Petruska AJ, Qiu FM, et al. 2015. 3D printed microtransporters: compound micromachines for spatiotemporally controlled delivery of therapeutic agents. Adv. Mater. 27:6644-50

7. Tottori S, Zhang L, Qiu F, Krawczyk KK, Franco-Obregón A, Nelson BJ. 2012. Magnetic helical micromachines: fabrication, controlled swimming, and cargo transport. Adv. Mater. 24:811-16

8. Huang T-Y, Qiu F, Tung H-W, Peyer KE, Shamsudhin N, et al. 2014. Cooperative manipulation and transport of microobjects using multiple helical microcarriers. $R S C A d v$. $4: 26771-76$

9. Peters C, Hoop M, Pane S, Nelson BJ, Hierold C. 2016. Degradable magnetic composites for minimally invasive interventions: device fabrication, targeted drug delivery, and cytotoxicity tests. Adv. Mater. 28:533-38

10. Zeeshan MA, Grisch R, Pellicer E, Sivaraman KM, Peyer KE, et al. 2014. Hybrid helical magnetic microrobots obtained by 3D template-assisted electrodeposition. Small 10:1284-88

11. Zhang L, Peyer KE, Nelson BJ. 2010. Artificial bacterial flagella for micromanipulation. $L a b$ Chip 10:2203-15 
12. Qiu F, Mhanna R, Zhang L, Ding Y, Fujita S, Nelson BJ. 2014. Artificial bacterial flagella functionalized with temperature-sensitive liposomes for controlled release. Sensor Actuat. B 196:676-81

13. Mhanna R, Qiu F, Zhang L, Ding Y, Sugihara K, et al. 2014. Artificial bacterial flagella for remote-controlled targeted single-cell drug delivery. Small 10:1953-57

14. Qiu F, Fujita S, Mhanna R, Zhang L, Simona BR, Nelson BJ. 2015. Magnetic helical microswimmers functionalized with lipoplexes for targeted gene delivery. Adv. Funct. Mater. 25:1666-71

15. Ania S, Qiu F, Mazza M, Kostarelos K, Nelson BJ. 2015. Controlled in vivo swimming of a swarm of bacteria-like microrobotic flagella. Adv. Mater. 27:2981-88

16. Kim S, Qiu F, Kim S, Ghanbari A, Moon C, et al. 2013. Fabrication and characterization of magnetic microrobots for three-dimensional cell culture and targeted transportation. $A d v$. Mater. 25:5863-68

17. Mourran A, Zhang H, Vinokur R, Moller M. 2017. Soft microrobots employing nonequilibrium actuation via plasmonic heating. Adv. Mater. 29:1604825

18. Snezhko A, Aranson IS. 2011. Magnetic manipulation of self-assembled colloidal asters. Nat. Mater. 10:698-703

19. Singh AV, Hosseinidoust Z, Park B-W, Yasa O, Sitti M. 2017. Microemulsion-based soft bacteria-driven microswimmers for active cargo delivery. ACS Nano 11:9759-69

20. Bartlett NW, Tolley MT, Overvelde JTB, Weaver JC, Mosadegh B, et al. 2015. A 3Dprinted, functionally graded soft robot powered by combustion. Science 349:161-65

21. Sato Y, Hiratsuka Y, Kawamata I, Murata S, Nomura SM. 2017. Micrometer-sized molecular robot changes its shape in response to signal molecules. Sci. Robot. 2:eaal3735

22. Reed EM, Wallace HR. 1965. Leaping locomotion by an insect-parasitic nematode. Nature 206:210-11

23. Jarrell KF, McBride MJ. 2008. The surprisingly diverse ways that prokaryotes move. Nat. Rev. Microbiol. 6:466-76

24. Tam D, Hosoi AE. 2011. Optimal kinematics and morphologies for spermatozoa. Phys. Rev. E 83 
25. Woolley DM, Crockett RF, Groom WDI, Revell SG. 2009. A study of synchronisation between the flagella of bull spermatozoa, with related observations. J. Exp. Biol. 212:221523

26. Darnton NC, Turner L, Rojevsky S, Berg HC. 2007. On torque and tumbling in swimming Escherichia coli. J. Bacteriol. 189:1756-64

27. Berg HC, Anderson RA. 1973. Bacteria swim by rotating their flagellar filaments. Nature $245: 380-82$

28. Elgeti J, Winkler RG, Gompper G. 2015. Physics of microswimmers-single particle motion and collective behavior: a review. Rep. Prog. Phys. 78:056601

29. Ozin GA, Manners I, Fournier-Bidoz S, Arsenault A. 2005. Dream nanomachines. Adv. Mater. 17:3011-18

30. Sengupta S, Ibele ME, Sen A. 2012. Fantastic voyage: designing self-powered nanorobots. Angew. Chem. Int. Ed. 51:8434-45

31. Peyer KE, Zhang L, Nelson BJ. 2013. Bio-inspired magnetic swimming microrobots for biomedical applications. Nanoscale 5:1259-72

32. Ehlers KM, Samuel ADT, Berg HC, Montgomery R. 1996. Do cyanobacteria swim using traveling surface waves? PNAS 93:8340-43

33. Shaevitz JW, Lee JY, Fletcher DA. 2005. Spiroplasma swim by a processive change in body helicity. Cell 122:941-45

34. Vollmers K, Frutiger DR, Kratochvil BE, Nelson BJ. 2008. Wireless resonant magnetic microactuator for untethered mobile microrobots. Appl. Phys. Lett. 92:144103

35. Frutiger DR, Vollmers K, Kratochvil BE, Nelson BJ. 2010. Small, fast, and under control: Wireless resonant magnetic micro-agents. Int. J. Robot. Res. 29:613-36

36. Tung HW, Maffioli M, Frutiger DR, Sivaraman KM, Pane S, Nelson BJ. 2014. Polymerbased wireless resonant magnetic microrobots. IEEE Trans. Robot. 30:26-32

37. Randhawa JS, Leong TG, Bassik N, Benson BR, Jochmans MT, Gracias DH. 2008. Pickand-place using chemically actuated microgrippers. J. Am. Chem. Soc. 130:17238-39

38. Bassik N, Brafman A, Zarafshar AM, Jamal M, Luvsanjav D, et al. 2010. Enzymatically triggered actuation of miniaturized tools. J. Am. Chem. Soc. 132:16314-17

39. Leong TG, Randall CL, Benson BR, Bassik N, Stern GM, Gracias DH. 2009. Tetherless thermobiochemically actuated microgrippers. PNAS 106:703-8 
40. Gultepe E, Yamanaka S, Laflin KE, Kadam S, Shim Y, et al. 2013. Biologic tissue sampling with untethered microgrippers. Gastroenterology 144:691-93

41. Gultepe E, Randhawa JS, Kadam S, Yamanaka S, Selaru FM, et al. 2013. Biopsy with thermally-responsive untethered microtools. Adv. Mater. 25:514-19

42. Malachowski K, Jamal M, Jin QR, Polat B, Morris CJ, Gracias DH. 2014. Self-folding single cell grippers. Nano Lett. 14:4164-70

43. Yim S, Gultepe E, Gracias DH, Sitti M. 2014. Biopsy using a magnetic capsule endoscope carrying, releasing, and retrieving untethered microgrippers. IEEE Trans. Bio-Med. Eng. $61: 513-21$

44. Diller E, Sitti M. 2014. Three-dimensional programmable assembly by untethered magnetic robotic micro-grippers. Adv. Funct. Mater. 24:4397-404

45. Qiu T, Lee TC, Mark AG, Morozov KI, Munster R, et al. 2014. Swimming by reciprocal motion at low reynolds number. Nat. Commun. 5:5119

46. Iss C, Ortiz G, Truong A, Hou YX, Livache T, et al. 2017. Fabrication of nanotweezers and their remote actuation by magnetic fields. Sci. Rep. 7:451

47. Kim S, Lee S, Lee J, Nelson BJ, Zhang L, Choi H. 2016. Fabrication and manipulation of ciliary microrobots with non-reciprocal magnetic actuation. Sci. Rep. 6:30713

48. Kim J, Chung SE, Choi SE, Lee H, Kim J, Kwon S. 2011. Programming magnetic anisotropy in polymeric microactuators. Nat. Mater. 10:747-52

49. Zhang JC, Diller E. 2016. Tetherless mobile micrograsping using a magnetic elastic composite material. Smart Mater. Struct. 25:11LT03

50. Lum GZ, Ye Z, Dong XG, Marvi H, Erin O, et al. 2016. Shape-programmable magnetic soft matter. PNAS 113:E6007-E15

51. Dreyfus R, Baudry J, Roper ML, Fermigier M, Stone HA, Bibette J. 2005. Microscopic artificial swimmers. Nature 437:862-65

52. Gao W, Sattayasamitsathit S, Manesh KM, Weihs D, Wang J. 2010. Magnetically powered flexible metal nanowire motors. J. Am. Chem. Soc. 132:14403-5

53. Pak OS, Gao W, Wang J, Lauga E. 2011. High-speed propulsion of flexible nanowire motors: Theory and experiments. Soft Matter 7:8169-81

54. Gao W, Manesh KM, Hua J, Sattayasamitsathit S, Wang J. 2011. Hybrid nanomotor: a catalytically/magnetically powered adaptive nanowire swimmer. Small 7:2047-51 
55. Li TL, Li JX, Zhang HT, Chang XC, Song WP, et al. 2016. Magnetically propelled fish-like nanoswimmers. Small 12:6098-105

56. Li TL, Li JX, Morozov KI, Wu ZG, Xu TL, et al. 2017. Highly efficient freestyle magnetic nanoswimmer. Nano Lett. 17:5092-98

57. Khalil ISM, Dijkslag HC, Abelmann L, Misra S. 2014. Magnetosperm: a microrobot that navigates using weak magnetic fields. Appl. Phys. Lett. 104:223701

58. Jang B, Gutman E, Stucki N, Seitz BF, Wendel-Garcia PD, et al. 2015. Undulatory locomotion of magnetic multilink nanoswimmers. Nano Lett. 15:4829-33

59. Mirkovic T, Foo ML, Arsenault AC, Fournier-Bidoz S, Zacharia NS, Ozin GA. 2007. Hinged nanorods made using a chemical approach to flexible nanostructures. Nat. Nanotechnol. 2:565-69

60. Purcell EM. 1977. Life at low Reynolds number. Am. J. Phys. 45:3-11

61. Ahmed D, Baasch T, Jang B, Pane S, Dual J, Nelson BJ. 2016. Artificial swimmers propelled by acoustically activated flagella. Nano Lett. 16:4968-74

62. Yoshizumi Y, Suzuki H. 2017. Self-propelled metal-polymer hybrid micromachines with bending and rotational motions. ACS Appl. Mater. Interfaces 9:21355-61

63. Jeong B, Kim SW, Bae YH. 2012. Thermosensitive sol-gel reversible hydrogels. Adv. Drug Deliv. Rev. 64:154-62

64. Kulkarni RV, Biswanath S. 2007. Electrically responsive smart hydrogels in drug delivery: a review. J. Appl. Biomater. Biomech. 5:125-39

65. Li H, Go G, Ko SY, Park JO, Park S. 2016. Magnetic actuated pH-responsive hydrogel-based soft micro-robot for targeted drug delivery. Smart Mater. Struct. 25:027001

66. Mohd Jani J, Leary M, Subic A, Gibson MA. 2014. A review of shape memory alloy research, applications and opportunities. Mater. Des. 56:1078-113

67. Clark AE, Restorff JB, Wun-Fogle M, Lograsso TA, Schlagel DL. 2000. Magnetostrictive properties of body-centered cubic Fe-Ga and Fe-Ga-Al alloys. IEEE Trans. Magn. 36:323840

68. Wang ZL, Song J. 2006. Piezoelectric nanogenerators based on zinc oxide nanowire arrays. Science 312:242-46

69. Holtz JH, Asher SA. 1997. Polymerized colloidal crystal hydrogel films as intelligent chemical sensing materials. Nature 389:829-32 
70. Geryak R, Tsukruk VV. 2014. Reconfigurable and actuating structures from soft materials. Soft Matter 10:1246-63

71. Erlanger BF, Kokowsky N, Cohen W. 1961. The preparation and properties of two new chromogenic substrates of trypsin. Arch. Biochem. Biophys. 95:271-78

72. Donnelly RF, Singh TRR, Alkilani AZ, McCrudden MTC, O’Neill S, et al. 2013. Hydrogelforming microneedle arrays exhibit antimicrobial properties: potential for enhanced patient safety. Int. J. Pharm 451:76-91

73. Fernandes R, Gracias DH. 2012. Self-folding polymeric containers for encapsulation and delivery of drugs. Adv. Drug Deliv. Rev. 64:1579-89

74. Hoffman AS. 2002. Hydrogels for biomedical applications. Adv. Drug Deliv. Rev. 54:3-12

75. Kim M, Jung B, Park JH. 2012. Hydrogel swelling as a trigger to release biodegradable polymer microneedles in skin. Biomaterials 33:668-78

76. Meenach SA, Shapiro JM, Hilt JZ, Anderson KW. 2013. Characterization of peg-iron oxide hydrogel nanocomposites for dual hyperthermia and paclitaxel delivery. J. Biomater. Sci. Polym. Ed. 24:1112-26

77. Hoare TR, Kohane DS. 2008. Hydrogels in drug delivery: progress and challenges. Polymer 49:1993-2007

78. Vashist A, Vashist A, Gupta YK, Ahmad S. 2014. Recent advances in hydrogel based drug delivery systems for the human body. J. Mater. Chem. B 2:147-66

79. Jeon SJ, Hauser AW, Hayward RC. 2017. Shape-morphing materials from stimuli-responsive hydrogel hybrids. Accounts Chem. Res. 50:161-69

80. Xu LZ, Shyu TC, Kotov NA. 2017. Origami and kirigami nanocomposites. ACS Nano 11:7587-99

81. Zou YQ, Yu K, Lai BFL, Brooks DE, Kizhakkedathu JN. 2013. Hemocompatible surfaces for blood-contacting applications. In Handbook of Biofunctional Surfaces, ed. W Knoll, pp. 923-59. Boca Raton, FL: CRC

82. Knop K, Hoogenboom R, Fischer D, Schubert US. 2010. Poly(ethylene glycol) in drug delivery: pros and cons as well as potential alternatives. Angew. Chem. Int. Ed. 49:6288-308

83. Zhao Q, Yang XX, Ma CX, Chen D, Bai H, et al. 2016. A bioinspired reversible snapping hydrogel assembly. Mater. Horiz. 3:422-28 
84. Deng H, Dong Y, Su J-W, Zhang C, Xie Y, et al. 2017. Bioinspired programmable polymer gel controlled by swellable guest medium. ACS Appl. Mater. Interfaces 9:30900-8

85. Ahn SK, Kasi RM, Kim SC, Sharma N, Zhou YX. 2008. Stimuli-responsive polymer gels. Soft Matter 4:1151-57

86. Li X, Serpe MJ. 2014. Understanding and controlling the self-folding behavior of poly ( $N$ isopropylacrylamide) microgel-based devices. Adv. Funct. Mater. 24:4119-26

87. Breger JC, Yoon C, Xiao R, Kwag HR, Wang MO, et al. 2015. Self-folding thermomagnetically responsive soft microgrippers. ACS Appl. Mater. Interfaces 7:3398-405

88. Hauser AW, Evans AA, Na JH, Hayward RC. 2015. Photothermally reprogrammable buckling of nanocomposite gel sheets. Angew. Chem. Int. Ed. 54:5434-37

89. Hu ZB, Zhang XM, Li Y. 1995. Synthesis and application of modulated polymer gels. Science 269:525-27

90. Fusco S, Sakar MS, Kennedy S, Peters C, Bottani R, et al. 2014. An integrated microrobotic platform for on-demand, targeted therapeutic interventions. Adv. Mater. 26:952-57

91. Yoon C, Xiao R, Park J, Cha J, Nguyen TD, Gracias DH. 2014. Functional stimuli responsive hydrogel devices by self-folding. Smart Mater. Struct. 23:094008

92. Malachowski K, Breger J, Kwag HR, Wang MO, Fisher JP, et al. 2014. Stimuli-responsive theragrippers for chemomechanical controlled release. Angew. Chem. Int. Ed. 53:8045-49

93. Stoychev G, Turcaud S, Dunlop JWC, Ionov L. 2013. Hierarchical multi-step folding of polymer bilayers. Adv. Funct. Mater. 23:2295-300

94. Therien-Aubin H, Wu ZL, Nie ZH, Kumacheva E. 2013. Multiple shape transformations of composite hydrogel sheets. J. Am. Chem. Soc. 135:4834-39

95. Wu ZL, Moshe M, Greener J, Therien-Aubin H, Nie ZH, et al. 2013. Three-dimensional shape transformations of hydrogel sheets induced by small-scale modulation of internal stresses. Nat. Commun. 4:1586

96. Huang HW, Sakar MS, Petruska AJ, Pane S, Nelson BJ. 2016. Soft micromachines with programmable motility and morphology. Nat. Commun. 7:12263

97. Huang HW, Chao Q, Sakar MS, Nelson BJ. 2017. Optimization of tail geometry for the propulsion of soft microrobots. IEEE Robot. Autom. Lett. 2:727-32

98. Wang W, Duan WT, Sen A, Mallouk TE. 2013. Catalytically powered dynamic assembly of rod-shaped nanomotors and passive tracer particles. PNAS 110:17744-49 
99. Palacci J, Sacanna S, Steinberg AP, Pine DJ, Chaikin PM. 2013. Living crystals of lightactivated colloidal surfers. Science 339:936-40

100. Kagan D, Balasubramanian S, Wang J. 2011. Chemically triggered swarming of gold microparticles. Angew. Chem. Int. Ed. 50:503-6

101. Solovev AA, Sanchez S, Schmidt OG. 2013. Collective behaviour of self-propelled catalytic micromotors. Nanoscale 5:1284-93

102. Thakur S, Kapral R. 2012. Collective dynamics of self-propelled sphere-dimer motors. Phys. Rev. E 85:026121

103. Magdanz V, Stoychev G, Ionov L, Sanchez S, Schmidt OG. 2014. Stimuli-responsive microjets with reconfigurable shape. Angew. Chem. Int. Ed. 53:2673-77

104. Palagi S, Mark AG, Reigh SY, Melde K, Qiu T, et al. 2016. Structured light enables biomimetic swimming and versatile locomotion of photoresponsive soft microrobots. Nat. Mater. 15:647-54

105. White TJ, Broer DJ. 2015. Programmable and adaptive mechanics with liquid crystal polymer networks and elastomers. Nat. Mater. 14:1087-98

106. Wani OM, Zeng H, Priimagi A. 2017. A light-driven artificial flytrap. Nat. Commun. $8: 15546$

107. Xiong XL, Wu CC, Zhou CS, Zhu GZ, Chen Z, Tan WH. 2013. Responsive DNA-based hydrogels and their applications. Macromol. Rapid. Commun. 34:1271-83

108. Hu YW, Kahn JS, Guo WW, Huang FJ, Fadeev M, et al. 2016. Reversible modulation of DNA-based hydrogel shapes by internal stress interactions. J. Am. Chem. Soc. 138:16112-19

109. Cangialosi A, Yoon C, Liu J, Huang Q, Guo J, et al. 2017. DNA sequence-directed shape change of photopatterned hydrogels via high-degree swelling. Science 357:1126-30

110. Wilson DA, Nolte RJM, van Hest JCM. 2012. Autonomous movement of platinum-loaded stomatocytes. Nat. Chem. 4:268-74

111. Peng F, Tu YF, van Hest JCM, Wilson DA. 2015. Self-guided supramolecular cargo-loaded nanomotors with chemotactic behavior towards cells. Angew. Chem. Int. Ed. 54:11662-65

112. van Rhee PG, Rikken RSM, Abdelmohsen LKEA, Maan JC, Nolte RJM, et al. 2014. Polymersome magneto-valves for reversible capture and release of nanoparticles. Nat. Commun. 5:5010 
113. Tu YF, Peng F, White PB, Wilson DA. 2017. Redox-sensitive stomatocyte nanomotors: destruction and drug release in the presence of glutathione. Angew. Chem. Int. Ed. 56:762024

114. Wu ZG, Wu YJ, He WP, Lin XK, Sun JM, He Q. 2013. Self-propelled polymer-based multilayer nanorockets for transportation and drug release. Angew. Chem. Int. Ed. 52:7000-3

115. Wu Z, Lin X, Wu Y, Si T, Sun J, He Q. 2014. Near-infrared light-triggered “on/off” motion of polymer multilayer rockets. ACS Nano 8:6097-105

116. Wu ZG, Lin XK, Zou X, Sun JM, He Q. 2015. Biodegradable protein-based rockets for drug transportation and light-triggered release. ACS Appl. Mater. Interfaces 7:250-55

117. Carlsen RW, Edwards MR, Zhuang J, Pacoret C, Sitti M. 2014. Magnetic steering control of multi-cellular bio-hybrid microswimmers. Lab Chip 14:3850-59

118. Sakar MS, Steager EB, Kim DH, Julius AA, Kim M, et al. 2011. Modeling, control and experimental characterization of microbiorobots. Int. J. Robot. Res. 30:647-58

119. Kojima M, Zhang ZH, Nakajima M, Ooe K, Fukuda T. 2013. Construction and evaluation of bacteria-driven liposome. Sensor Actuat. B 183:395-400

120. Xi JZ, Schmidt JJ, Montemagno CD. 2005. Self-assembled microdevices driven by muscle. Nat. Mater. 4:180-84

121. Taherkhani S, Mohammadi M, Daoud J, Martel S, Tabrizian M. 2014. Covalent binding of nanoliposomes to the surface of magnetotactic bacteria for the synthesis of self-propelled therapeutic agents. ACS Nano 8:5049-60

122. Zhuang J, Sitti M. 2016. Chemotaxis of bio-hybrid multiple bacteria-driven microswimmers. Sci. Rep. 6:32135

123. Singh AV, Sitti M. 2016. Patterned and specific attachment of bacteria on biohybrid bacteria-driven microswimmers. Adv. Healthc. Mater. 5:2325-31

124. Carlsen RW, Sitti M. 2014. Bio-hybrid cell-based actuators for microsystems. Small 10:3831-51

125. Feinberg AW. 2015. Biological soft robotics. Annu. Rev. Biomed. Eng. 17:243-65

126. Feinberg AW, Feigel A, Shevkoplyas SS, Sheehy S, Whitesides GM, Parker KK. 2007. Muscular thin films for building actuators and powering devices. Science 317:1366-70

127. Kim J, Park J, Yang S, Baek J, Kim B, et al. 2007. Establishment of a fabrication method for a long-term actuated hybrid cell robot. Lab Chip 7:1504-8 
128. Nawroth JC, Lee H, Feinberg AW, Ripplinger CM, McCain ML, et al. 2012. A tissueengineered jellyfish with biomimetic propulsion. Nat. Biotechnol. 30:792-97

129. Park SJ, Gazzola M, Park KS, Park S, Di Santo V, et al. 2016. Phototactic guidance of a tissue-engineered soft-robotic ray. Science 353:158-62

130. Chan V, Park K, Collens MB, Kong H, Saif TA, Bashir R. 2012. Development of miniaturized walking biological machines. Sci. Rep. 2:857

131. Williams BJ, Anand SV, Rajagopalan J, Saif MTA. 2014. A self-propelled biohybrid swimmer at low Reynolds number. Nat. Commun. 5:3081

132. Sakar MS, Neal D, Boudou T, Borochin MA, Li YQ, et al. 2012. Formation and optogenetic control of engineered 3D skeletal muscle bioactuators. Lab Chip 12:4976-85

133. Raman R, Cvetkovic C, Uzel SGM, Platt RJ, Sengupta P, et al. 2016. Optogenetic skeletal muscle-powered adaptive biological machines. PNAS 113:3497-502

134. Cvetkovic C, Raman R, Chan V, Williams BJ, Tolish M, et al. 2014. Three-dimensionally printed biological machines powered by skeletal muscle. PNAS 111:10125-30

6a. Donald BR, Levey CG, McGray CD, Paprotny I, Rus D. 2006. An untethered, electrostatic, globally controllable mems micro-robot. J. Microelectromech. S. 15: 1-15

Figures

Figure 1:

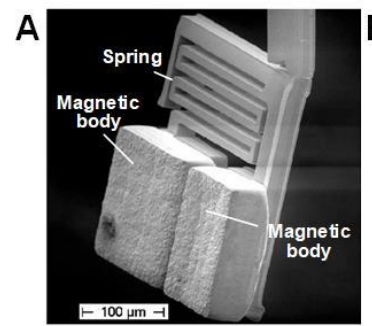

C

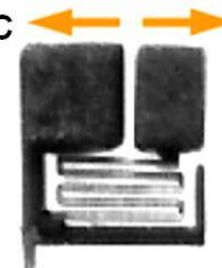

Fully open
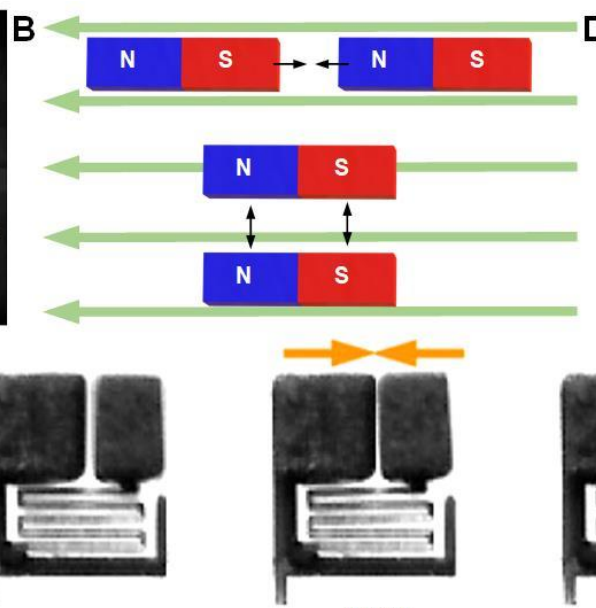

Impact
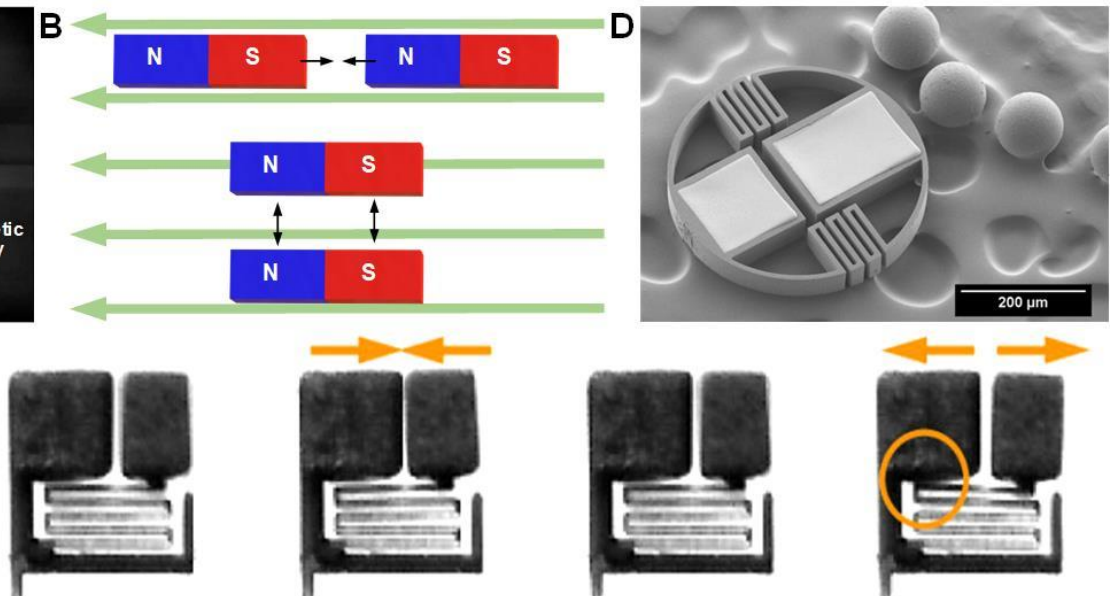

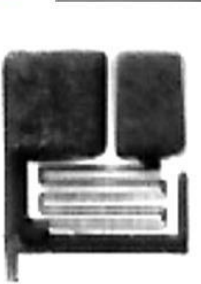

t

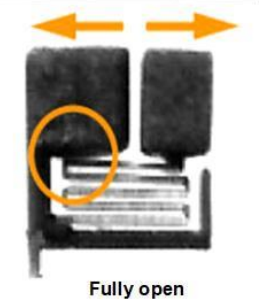

Fully open 
Figure 2:

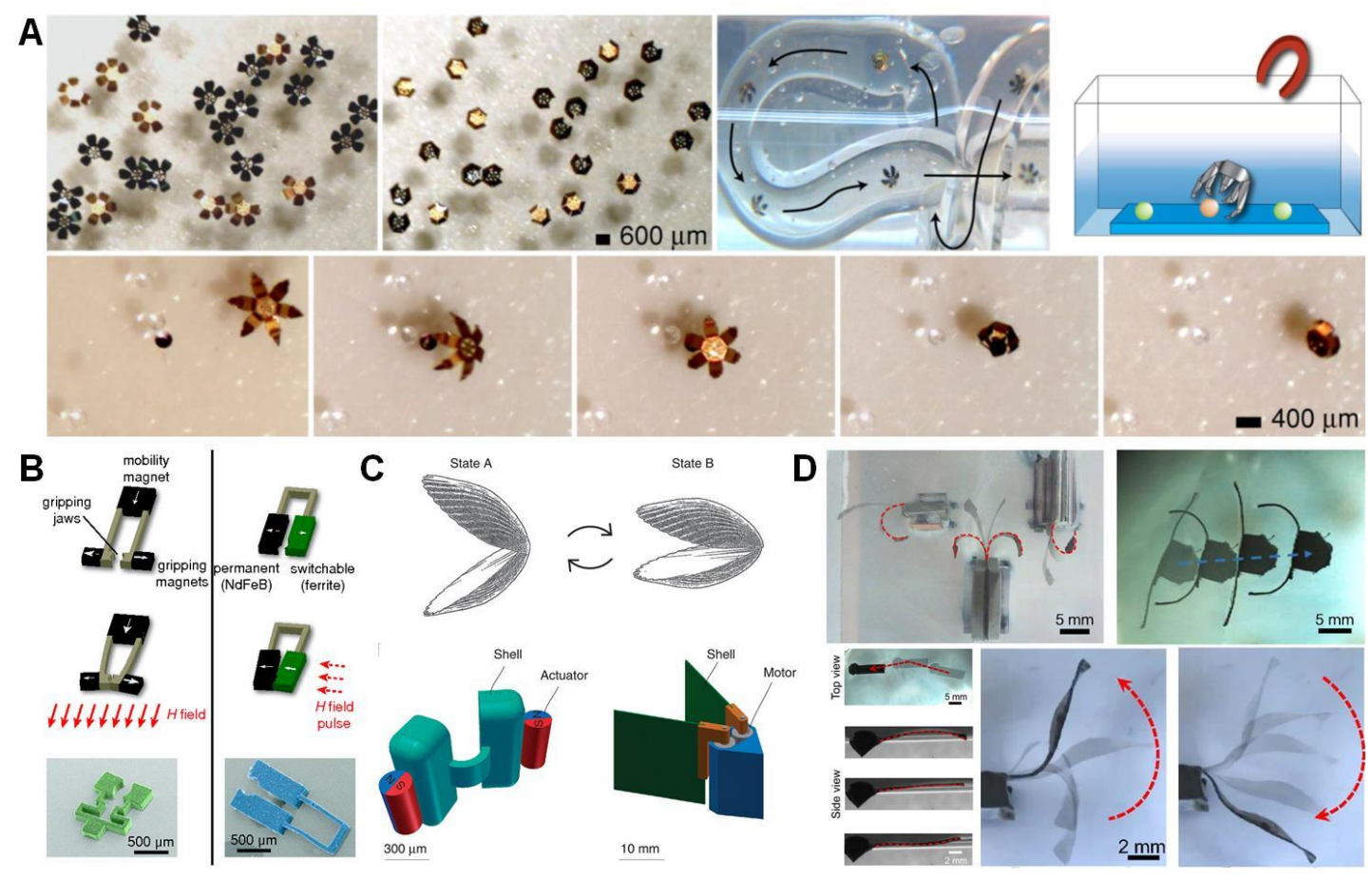

Figure 3: 


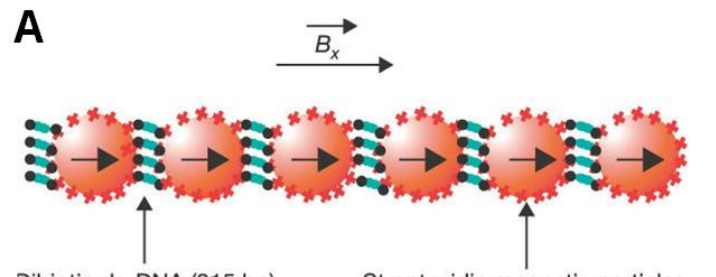

Dibiotin ds-DNA (315 bp) Streptavidin magnetic particles C
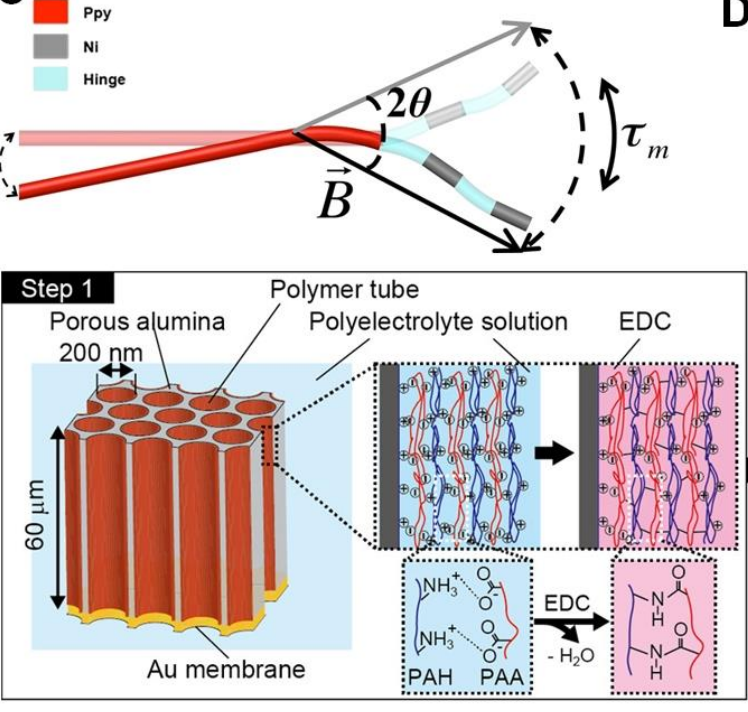

Step $1 \quad$ Polymer tube

Step 3

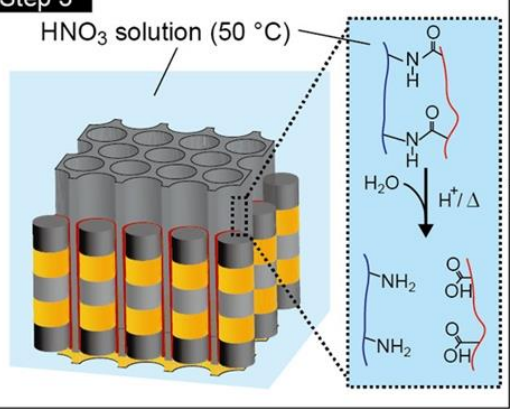

B
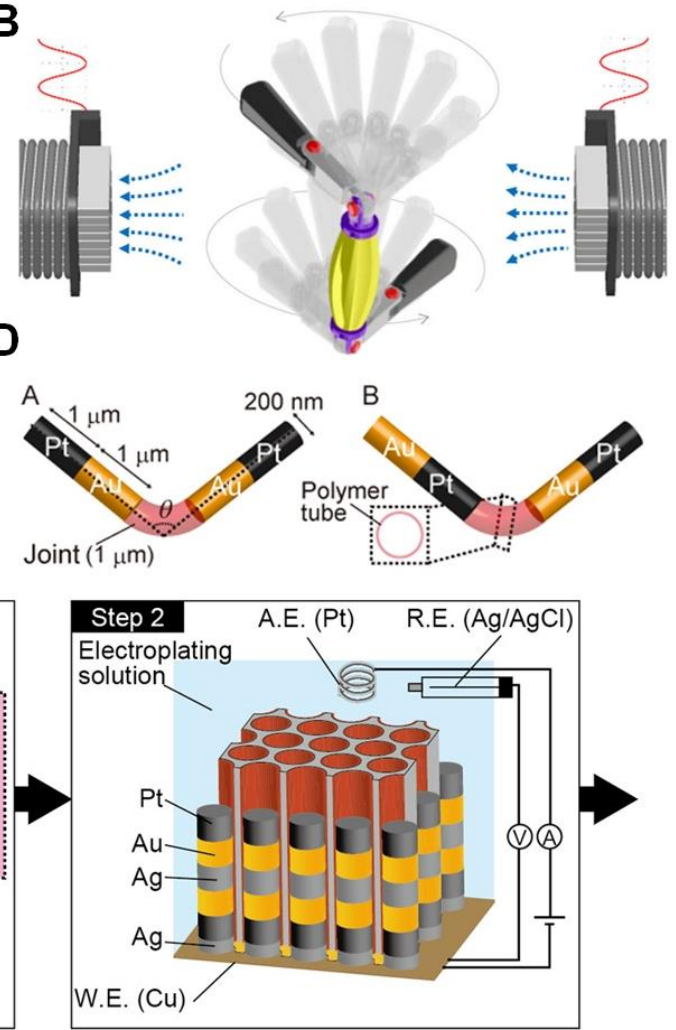

W.E. (Cu)

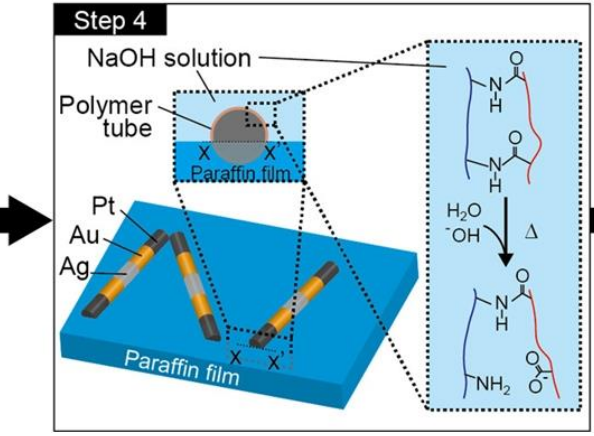

Step 5

$\mathrm{H}_{2} \mathrm{O}_{2}$ solution

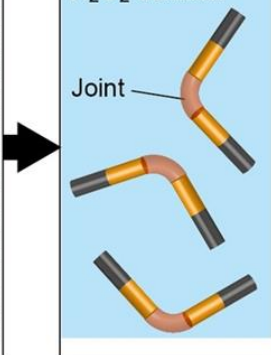


Figure 4:

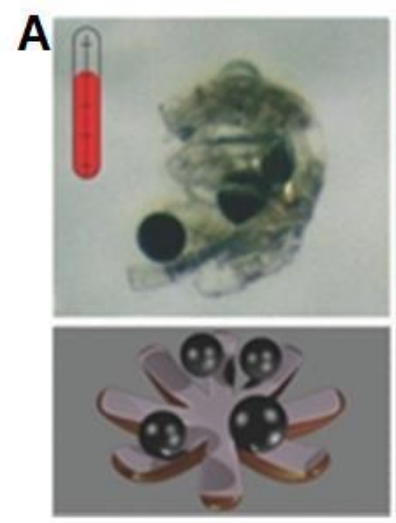

B
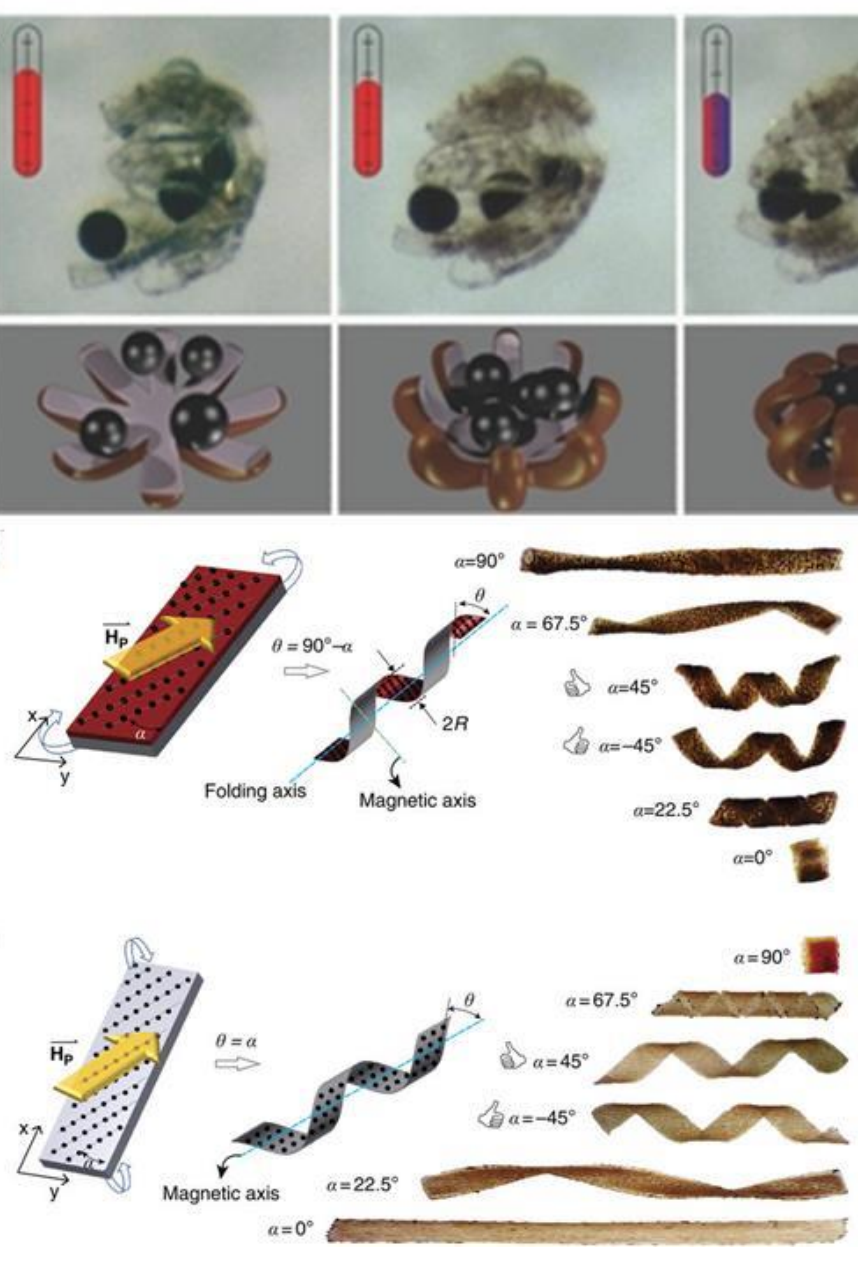
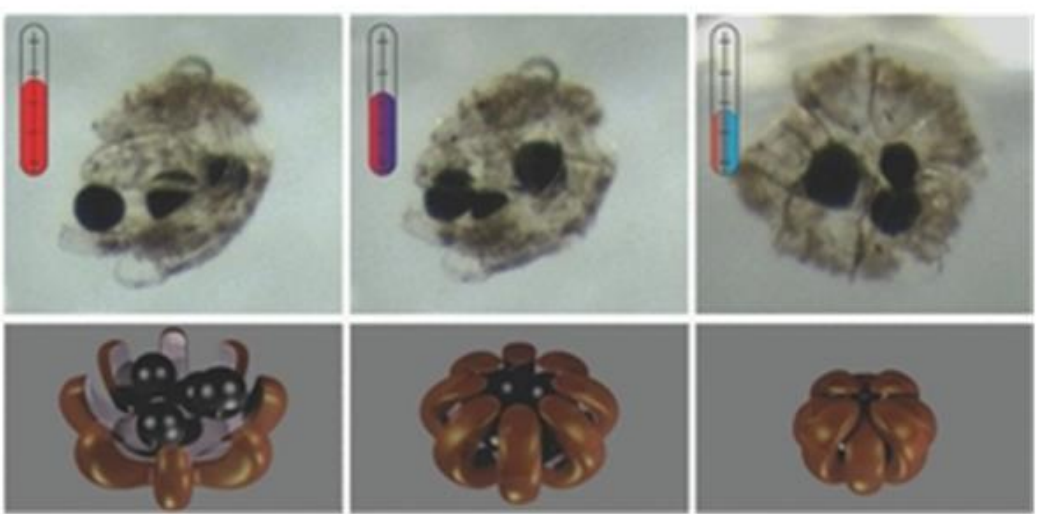

C
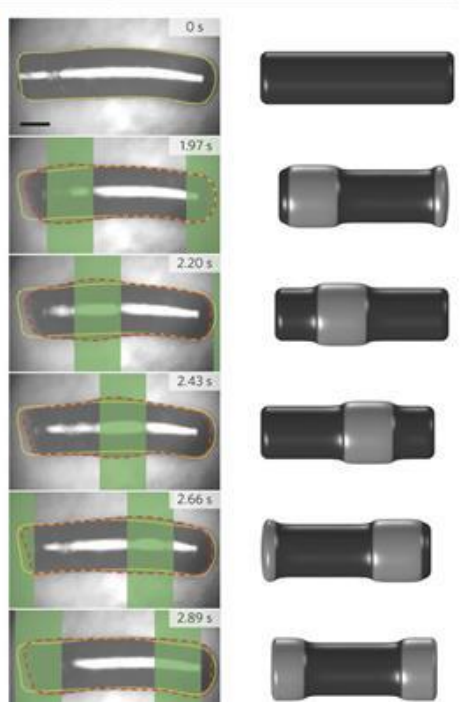
Figure 5:
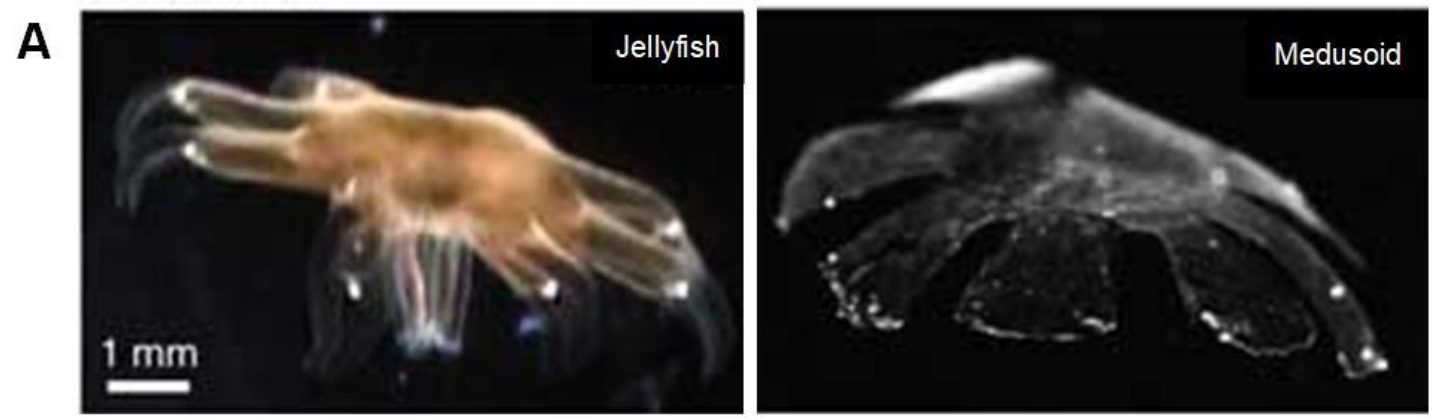

B ARTIFICIAL RAY
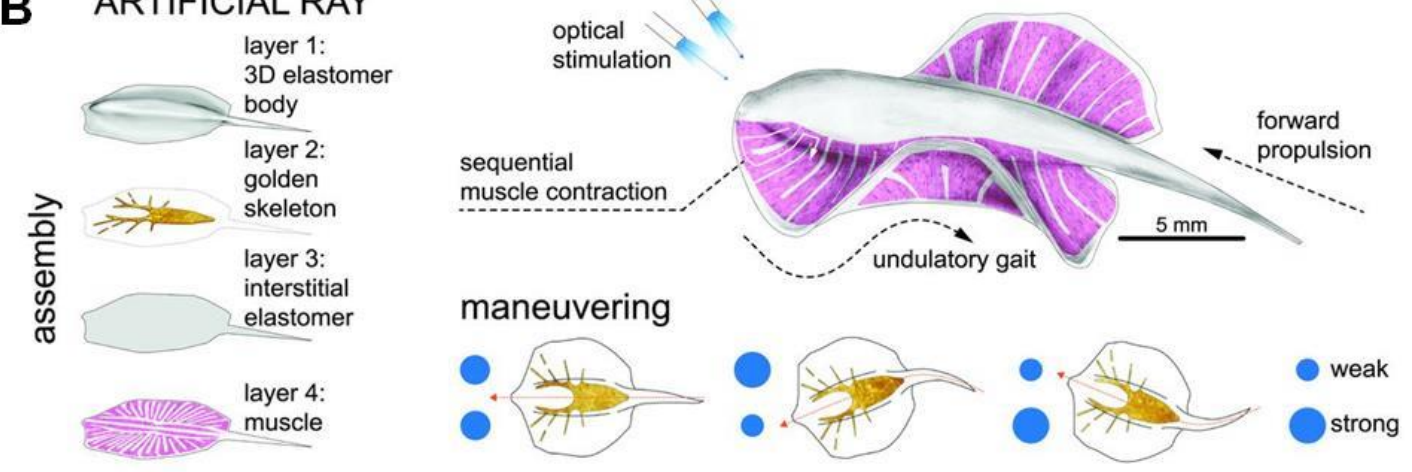

C
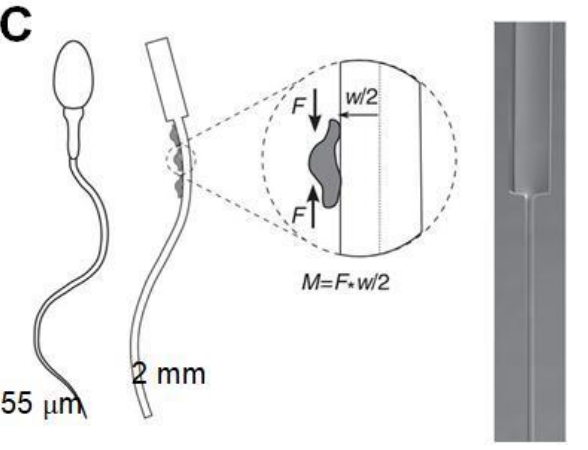

D

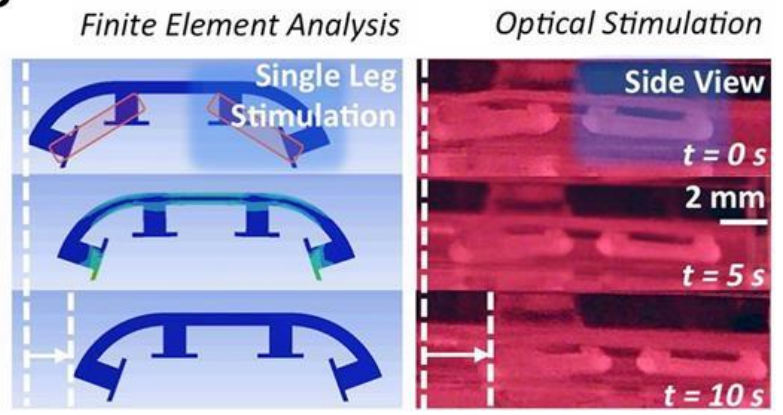

